\title{
Wedged double Wollaston, a device for single shot polarimetric measurements
}

\author{
E. Oliva ${ }^{1}$ \\ Osservatorio Astrofisico di Arcetri, Largo E. Fermi 5, I-50125 Firenze, Italy
}

Received September 5; accepted October 21, 1996

\begin{abstract}
Simultaneous measurements of the polarized flux at angles 0, 45, 90 and 135 degrees can be performed by inserting a simple combination of two Wollaston prisms and two wedges (cf. Fig. 1) in the pupil plane of a camera/spectrometer multimode astronomical instrument. The first three elements of the Stokes vector can be therefore determined from data taken at the same time and without any need of rotating $\lambda / 2$ plates or other moving elements which could be difficult to incorporate in multimode astronomical instruments.

This paper describes the working principles of the wedged double Wollaston and presents detailed parameters for prism combinations optimized for imagingpolarimetry and spectro-polarimetry at "visual" (0.3$1.0 \mu \mathrm{m})$ and near IR $(1.0-2.5 \mu \mathrm{m})$ wavelengths.

The devices described here could significantly simplify the implementation of imaging and spectro-polarimetric modes in astronomical multimode focal reducers.
\end{abstract}

Key words: instrumentation: miscellaneous, instrumentation: polarimeters

\section{Introduction}

Wollaston prisms are extensively used in focal plane astronomical instruments working at visual, and recently also at IR wavelengths (e.g. Packham et al. 1996). Their main practical advantage is that they allow a straightforward implementation of a polarimetric mode in multimode imager/spectrometers because a Wollaston prism inserted in the pupil plane of a focal reducer produces simultaneous images in two perpendicular polarization states on different parts of the array (see e.g. Fig. 3 of Fosbury et al. 1989). However, at least two separate measurements with the polarization axis rotated by $45^{\circ}$ are required to derive the first 3 elements of the Stokes vector, i.e. to determine the polarization degree and angle.

The most common and reliable method to complete the polarimetric measurement is to use a rotating $\lambda / 2$ plate before the Wollaston to modify the orientation of the polarization axis relative to the prism. This well known system may be difficult to implement in cryogenic instruments working in the IR for the following reasons.

- A rotating $\lambda / 2$ retarder inside the cold dewar requires a complex mechanical design, especially in a multimode instrument where a mechanism for inserting/removing the $\lambda / 2$ plate from the beam is also necessary.

- Adding a $\lambda / 2$ plate before the dewar window and telescope focal plane may significantly increase the thermal background and produce ghosts.

A simpler method could be obtaining measurements at different position angles, i.e. rotate the whole instrument with respect to the telescope axis. This could be an effective solution for imaging-polarimetry but cannot be used for long slit spectro-polarimetry.

A convenient alternative could be a fixed device which simultaneously produces polarized images at 0, 45, 90 and 135 degrees on four different parts of the array yielding therefore imaging or spectro-polarimetric measurements from a single exposure and without moving anything. This paper describes a simple combination of two Wollastons and two prisms (wedges) which satisfies the above requirements. Parameters of devices optimized for imaging and spectro-polarimetry at visual and IR wavelengths are also presented in Sect. 3.

\section{The wedged double Wollaston (WeDoWo)}

Figure 1 is a sketch of the device which consists of two wedges which split the pupil image and deviate all rays above/below the optical axis thus preventing vignetting at the interface between the two systems of prisms. The rays than enter into the two Wollastons which have crystal axis at $45^{\circ}$, and emerge at 4 different angles with relative intensities depending on the polarization angle and degree of the input light. The four images on the array correspond therefore to measurements performed with polarizers at 0 , 90, 45 and 135 degrees, i.e. the parameters needed to derive the first 3 elements of the Stokes vector (e.g. Shurcliff 1962). 

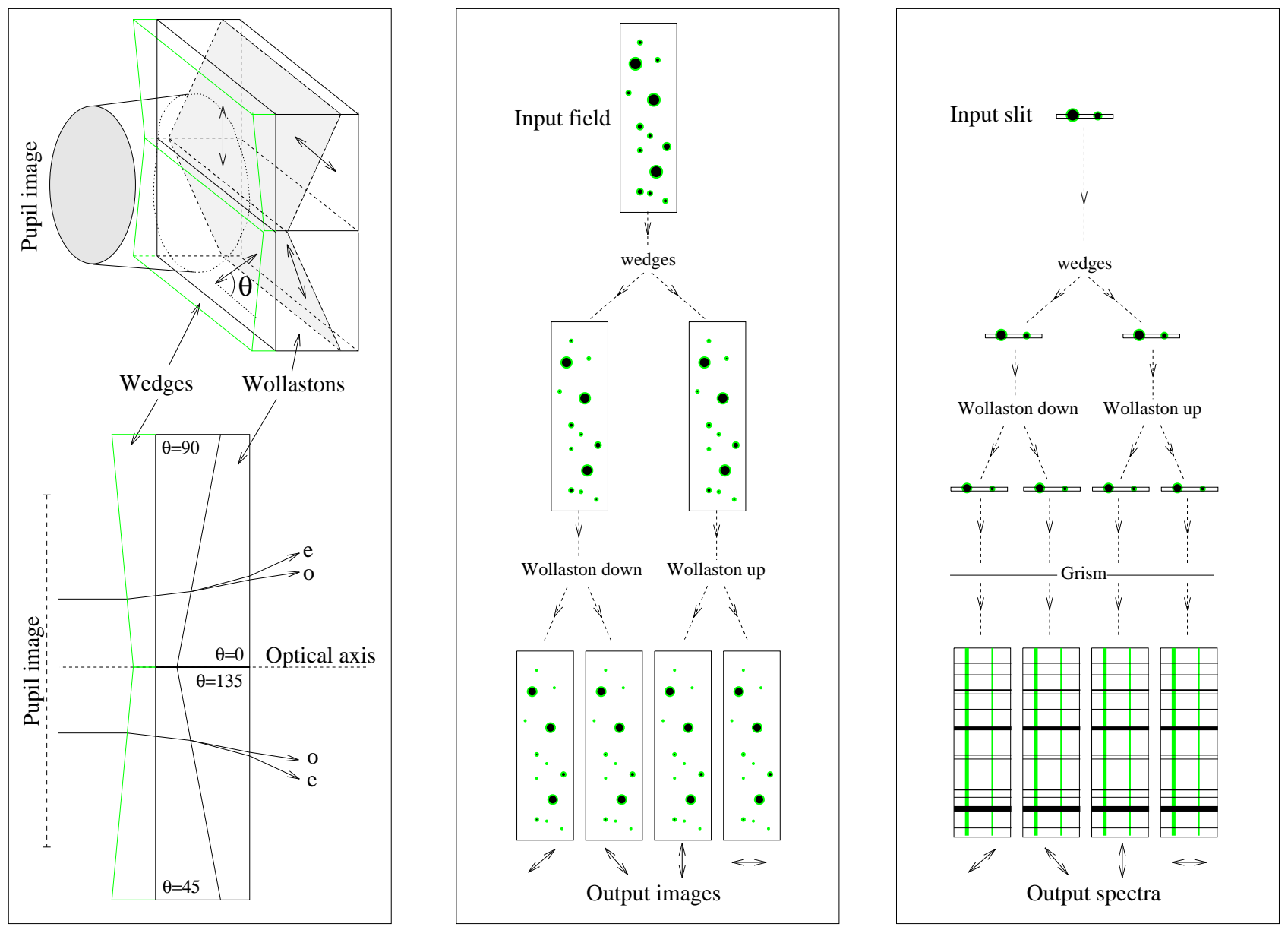

Fig. 1. Left: schematic representation of a WeDoWo device and ray-tracing. To prevent vignetting at the prisms interface the wedge angle is chosen to ensure that all rays (including those from the field edges) always travel above/below the optical axis. Center: illustration of how this device creates four polarized images (at 0, 90, 45 and 135 degrees) of a stellar field.

Right: illustration of how the WeDoWo creates four long slit polarized spectra (at 0, 90, 45 and 135 degrees) of two stars

The wedges and the Wollaston prisms are not necessarily separated elements, i.e. the wedge could be obtained by simply cutting the entrance face of the first Wollaston prism at a suitable angle. This simpler solution could be useful in cases where lateral chromatism is not a crucial issue (Sect. 3.1).

From the practical point of view, integrating a WeDoWo inside an instrument is as simple as using Wollastons, i.e. the device can be mounted in the filter or grism wheels of any focal reduced with a corrected, parallel pupil image. The only difference is that the input field mask should be designed taking into account that the WeDoWo produces four images.

\section{Practical applications}

\subsection{Spectro-polarimetry}

The crucial requirement for spectro-polarimetry with typical focal reducers is that the polarizing element should be as thin as possible to fit in the filter wheel, this implies that the Wollaston crystals should have a large birefringence.

As lateral chromatism is relatively little important in this mode, convenient (and probably the cheapest) materials are therefore $\mathrm{CaCO}_{3}$ and $\mathrm{LiNbO}_{3}$ for visual and IR instruments, respectively. The latter has $\Delta n=n_{\mathrm{e}}-n_{\mathrm{o}} \simeq$ -0.077 and is transparent from $5000 \AA$ to beyond $4 \mu \mathrm{m}$ while Calcite has $\Delta n \simeq-0.17$ and is transparent from below $3000 \AA$ to about $2.0 \mu \mathrm{m}$ (Oliva et al. 1996).

The entrance wedge can be most conveniently integrated in each Wollaston by cutting the entrance face of the first prism at a suitable angle (cf. Fig. 2). A consequence of this choice is that the output images suffer by different lateral chromatism and the outer spectra are much more distorted than the inner one's (cf. Fig. 3), but this can be easily taken into account during data reduction. Note that using separate quasi-achromatic wedges would give no practical advantage because the spectra are always distorted although symmetrical, i.e. the chromatism of the Wollaston cannot be corrected by the wedge. 

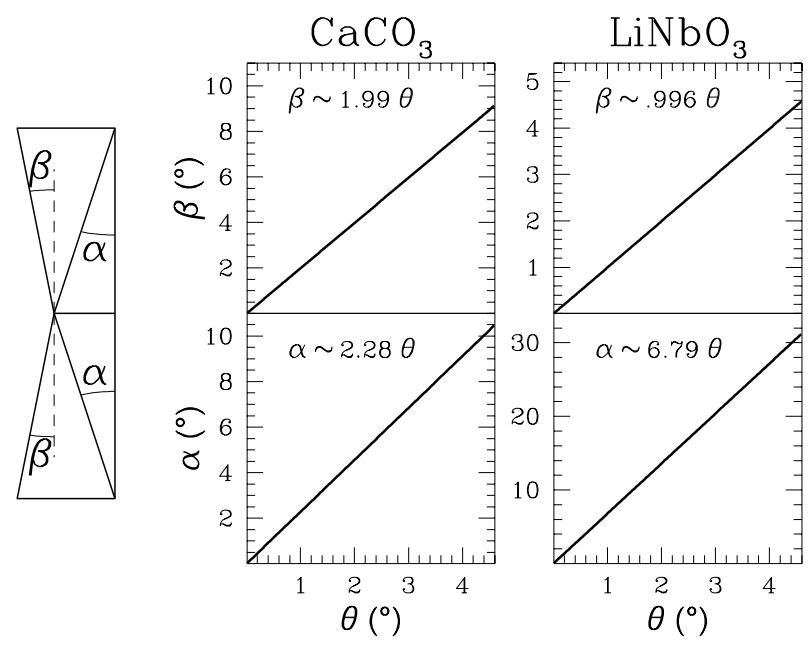

Fig. 2. Left: schematic representation of a thin WeDoWo device useful for spectro-polarimetry at visual $\left(\mathrm{CaCO}_{3}\right.$ prisms $)$ and IR ( $\mathrm{LiNbO}_{3}$ prisms) wavelengths.Center, right: values of the $\alpha$ and $\beta$ angles necessary to create four non-overlapping images of a slit. The parameter $\theta$ is the angle projected by the slit length onto the pupil image (Eq. 1). All computations are based on room temperature refractive indices of $\mathrm{CaCO}_{3}$ (Bennet 1995) while those of $\mathrm{LiNbO}_{3}$ are at $77 \mathrm{~K}$ using refraction indices and thermo-optic coefficients from Smith et al. (1976)
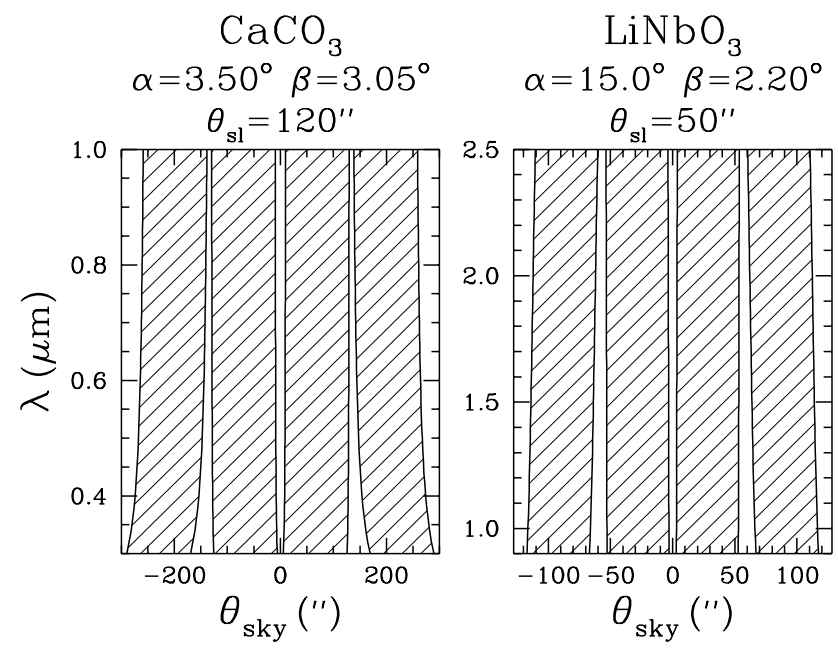

Fig. 3. Simulated spectra using thin WeDoWo devices, the distortion of the dispersed slit images is a consequence of the lateral chromatism of the wedges and Wollastons. The Wollaston and wedge angles $\alpha, \beta$ (cf. Fig. 2) and the slit lengths are chosen adopting

$D_{\text {tel }}=3.5 \mathrm{~m}, D_{\mathrm{p}}=7.6 \mathrm{~cm}$, field-of-view $=10^{\prime} \times 10^{\prime}$

$D_{\text {tel }}=3.5 \mathrm{~m}, D_{\mathrm{p}}=2.2 \mathrm{~cm}$, field-of-view $=4.3^{\prime} \times 4.3^{\prime}$

for the visual and infrared instrument, respectively
The angles $\alpha$ and $\beta$ required to create four nonoverlapping images of a slit of a given length are plotted in Fig. 2 as a function of the projected slit length $\theta$

$$
\left(\frac{\theta}{\text { degrees }}\right)=2.78\left(\frac{\theta_{\text {sky }}}{100^{\prime \prime}}\right)\left(\frac{D_{\text {tel }}}{1 \mathrm{~m}}\right)\left(\frac{D_{\mathrm{p}}}{1 \mathrm{~cm}}\right)^{-1}
$$

where $\theta_{\text {sky }}$ (sky projected angles) is the slit length, $D_{\text {tel }}$ is the telescope diameter and $D_{\mathrm{p}}$ is the diameter of the pupil image. Using the parameters of Fig. 2 the slit images are well spaced at all useful wavelengths, i.e. $0.3-1.0$ and $0.9-2.5 \mu \mathrm{m}$ for $\mathrm{CaCO}_{3}$ and $\mathrm{LiNbO}_{3}$, respectively. The four spectra are contained within the array if the sky projected field of view of the detector is $\geq 5$ times the slit length $\theta_{\text {sky }}$.

Representative examples of dispersed slit images produced by thin WeDoWo devices coupled with grisms (cf. right panel of Fig. 1) are displayed in Fig 3 where the strong lateral chromatism of $\mathrm{CaCO}_{3}$ at $\lambda<4000 \AA$ is particularly evident. The instrument parameters adopted in Fig. 3 are appropriate for the visual low dispersion spectrograph (LDS) and for the near infrared cameraspectrometer (NICS) of the Italian $3.5 \mathrm{~m}$ telescope TNG (Conconi 1992; Oliva \& Gennari 1995).

\subsection{Imaging-polarimetry}

Lateral chromatism of the Wollaston is a crucial issue for imaging-polarimetry because this parameter defines the maximum width of the field which can be reimaged without introducing a too strong image elongation. It is therefore convenient to manufacture the Wollastons using crystals with very low variation of birefringence with wavelengths, and the best materials which can be found on the market are probably $\mathrm{MgF}_{2}$ and $\mathrm{LiYF}_{4}$ (YLF) for visual and IR instruments, respectively. The latter is a synthetic compound developed for non linear applications with very interesting optical and mechanical properties. It is a tough, non-hygroscopic crystal transparent from $2000 \AA$ to $6 \mu \mathrm{m}$ and with a low refractive index, $n_{\mathrm{o}} \simeq 1.45$. The thermo-optic coefficients are very small, $\mathrm{d} n_{\mathrm{o}} / \mathrm{d} T \simeq-110^{-6}, \mathrm{~d} n_{\mathrm{e}} / \mathrm{d} T \simeq-310^{-6} \mathrm{~K}^{-1}$ (Barnes \& Gettemy D.J. 1980). YLF prisms are only slightly deformed when cooled because the thermal expansion coefficients along the two crystal axis are very similar, 13 $10^{-6} \mathrm{~K}^{-1} \| a$ and $810^{-6} \mathrm{~K}^{-1} \| c$ (Tropf et al. 1995). YLF is twice more birefringent than $\mathrm{MgF}_{2}(\Delta n \simeq 0.022)$ and, most important, is a factor $>2$ less chromatic than $\mathrm{MgF}_{2}$ in the $J, H, K$ IR photometric bands (for a detailed discussion of IR birefringent materials see also Oliva et al. 1996).

As both materials have low birefringence, relatively large Wollaston angles $\alpha$ are therefore required to obtain useful separations, i.e. the prisms are thick. This should not be a problem however because the WeDoWo devices can be mounted in the grism wheel which is usually designed to accommodate thick optical elements. 

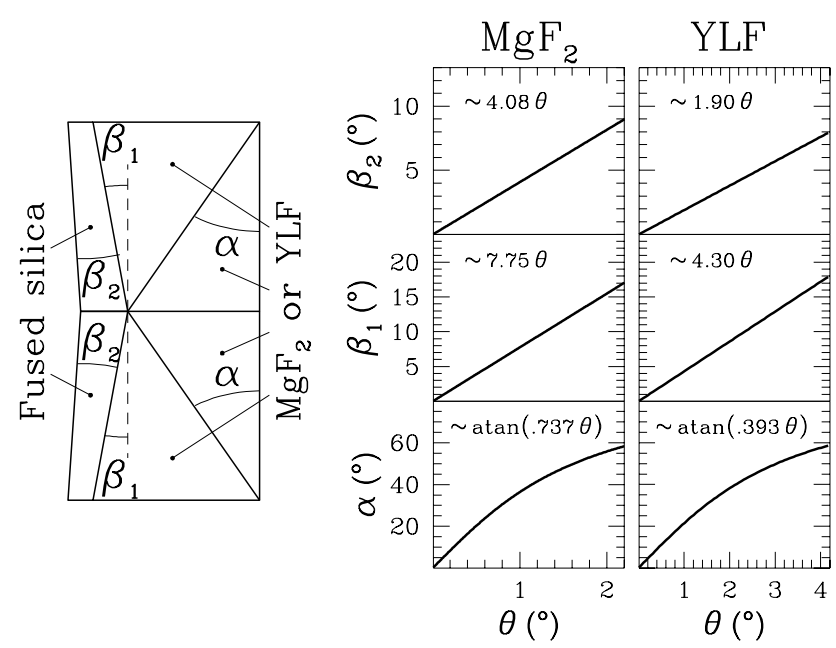

Fig. 4. Left: schematic representation of a WeDoWo device useful for imaging-polarimetry at visual $\left(\mathrm{MgF}_{2}\right.$ prisms $)$ and IR (YLF prisms) wavelengths, the prism of fused silica is used to achromatize the deviation of the wedge.

Center, right: values of the $\alpha, \beta_{1}$ and $\beta_{2}$ angles necessary to create four non-overlapping images of a field of view whose width projected onto the pupil image is $\theta$ (Eq. 1). All computations are based on room temperature refractive indices of $\mathrm{MgF}_{2}$ (Dodge 1980) while those of $\mathrm{LiYF}_{4}$ (YLF) are at $77 \mathrm{~K}$ (see text, Sect. 3.2)

The lateral chromatism of the wedge must be small compared to that of the Wollaston. This requirement could be strictly satisfied by manufacturing the wedge using two or more prisms of materials with different dispersions. A simpler solution is however that described in Fig. 4 and which takes advantage of the fact that both $\mathrm{MgF}_{2}$ and YLF are low dispersion optical materials (i.e. good "crowns") which can be coupled with fused silica (a cheap "flint" transparent at all $\lambda$ 's of interest) to produce wedges with very low chromatism.

The angles $\alpha, \beta_{1}$ and $\beta_{2}$ required to create four quasiachromatic non-overlapping images of a field of a given width are plotted in Fig. 4 as a function of the projected width of the field of view $\theta$ (Eq. 1). The lateral chromatism is virtually the same of that of a standard Wollaston and the largest image elongation (sky projected angles) for exposures taken through broad band astronomical filters is

$\epsilon(U)=\theta_{\text {sky }} / 110 ; \quad \epsilon(K)=\theta_{\text {sky }} / 220$

for the visual and IR WeDoWo, respectively.

Table 1 is a list of prisms angles and image elongation for WeDoWo devices designed using the instrumental parameters of LDS and NICS (cf. end of Sect. 3.1). The width of the input field of view is chosen to ensure that the image elongation is less than one pixel in all photometric bands.
Table 1. Parameters of devices for imaging-polarimetry ${ }^{(1)}$

\begin{tabular}{|lc|ll|}
\hline \multicolumn{2}{|l|}{ Visual WeDoWo $\left(\mathrm{MgF}_{2}\right)$} & \multicolumn{2}{|c|}{ IR WeDoWo $(\mathrm{YLF})$} \\
\hline$\alpha\left(^{\circ}\right)$ & 18.0 & $\alpha\left(^{\circ}\right)$ & 41.0 \\
$\beta_{1}\left({ }^{\circ}\right)$ & 3.41 & $\beta_{1}\left({ }^{\circ}\right)$ & 9.50 \\
$\beta_{2}\left({ }^{\circ}\right)$ & 1.80 & $\beta_{2}\left({ }^{\circ}\right)$ & 4.20 \\
$\theta_{\text {sky }}\left({ }^{\prime \prime}\right)$ & 35 & $\theta_{\text {sky }}\left({ }^{\prime \prime}\right)$ & 50 \\
$\epsilon(U, B)\left({ }^{\prime \prime}\right)$ & 0.31 & $\epsilon(J)\left(^{\prime \prime}\right)$ & 0.18 \\
$\epsilon(V)\left({ }^{\prime \prime}\right)$ & 0.20 & $\epsilon(H)\left(^{\prime \prime}\right)$ & 0.03 \\
$\epsilon(R, I)\left({ }^{\prime \prime}\right)$ & 0.11 & $\epsilon(K)\left({ }^{\prime \prime}\right)$ & 0.23 \\
\hline
\end{tabular}

(1) Prism angles $\alpha, \beta_{1}$ and $\beta_{2}$ (Fig. 4) are computed assuming $D_{\text {tel }}=3.5 \mathrm{~m}, D_{\mathrm{p}}=7.6 \mathrm{~cm}$

$D_{\text {tel }}=3.5 \mathrm{~m}, D_{\mathrm{p}}=2.2 \mathrm{~cm}$

for the visual and infrared instrument, respectively. The maximum width of the input field is $\theta_{\text {sky }}$ while $\epsilon$ is the image elongation for imaging through the photometric filters.

\section{Conclusions}

Single shot polarimetric measurements can be performed using wedged double Wollaston, i.e. a combination of 2 wedges and 2 Wollaston prisms. The WeDoWo devices described in this paper could significantly simplify the implementation of imaging and spectro-polarimetric modes in astronomical multimode focal reducers.

\section{References}

Bennet J.M., 1995, Handbook of Optics $2^{\text {nd }}$ edition. In: Bass M. (ed.). McGraw-Hill (New York), Vol. II, Chapter 3

Barnes N.P., Gettemy D.J., 1980, J. Opt. Soc. Am. 70, 1244

Conconi P., 1992, TNG Instrument Plan: a progress report. In: Fusi Pecci F., Stirpe G.M. (eds.) Astronomical Observatory of Bologna, p. 101

Dodge M.J., 1980, Appl. Opt. 23, 1980

Fosbury R., Di Serego Alighieri S., Tadhunter C.N., 1989, The Messenger 57, 49

Oliva E., Gennari S., 1995, A\&AS 114, 179

Oliva E., Gennari S., Vanzi L., Caruso A., Ciofini M., 1996, A\&AS (in press) (Arcetri preprint Nos. 24/96)

Packham C., Hough J.H., Young S., Chrysostomou A., Bailey J.A., Axon D.J., Ward M.J., 1996, MNRAS 278, 406

Shurcliff W.A., 1962, Polarized light. Harvard Univ. Press, Cambridge

Smith D.S., Riccius H.D., Edwin R.P., 1976, Opt. Commun. 17,332

Tropf W.J., Thomas M.E., Harris T.J., 1995, Handbook of Optics $2^{\text {nd }}$ edition. In: Bass M. (ed.). McGraw-Hill (New York), Vol. II, p. 33 